\title{
Sub-Doppler UV spectroscopy by resonance-enhanced two-photon ionization: the structure of the benzene- ${ }^{20,22} \mathrm{Ne}$ cluster
}

\author{
Th. Weber, E. Riedle, H.J. Neusser and E.W. Schlag \\ Institut für Physikalische und Theoretische Chemie, Technische Universität München, \\ Lichtenbergstraße 4, W-8046 Garching, (Germany)
}

(Received 10 December 1990)

\begin{abstract}
In this work it is demonstrated that mass selected multiphoton ionization is a powerful technique for high resolution spectroscopy, isotope cluster separation and investigation of the structure of van der Waals clusters. The rotationally resolved UV spectra of the benzene- ${ }^{20} \mathrm{Ne}$ and benzene $-{ }^{22} \mathrm{Ne}$ clusters are selectively measured in a natural isotopic mixture of benzene and benzene-Ne clusters in a cooled supersonic jet. The analysis of these spectra yields accurate values for the rotational constants of both species. From this data it is found that the $\mathrm{Ne}$ atom is located on the $C_{6}$ rotational axis of the benzene ring at an average distance of $3.46 \AA$ with a slight difference for the two isotopes. This distance decreases by $40 \mathrm{~m} \AA$ when benzene is electronically excited. The influence of the large amplitude van der Waals vibrations on the average bond length is discussed.
\end{abstract}

\section{INTRODUCTION}

In the last decade, the application of multiphoton processes in molecular spectroscopy has provided a wealth of information on molecular excited states [1]. In general, selection rules for multiphoton absorption are different from those of one-photon absorption. As a consequence, two-photon spectroscopy of large molecules yielded new information on hitherto unknown vibrational and electronic states, which cannot be accessed in one-photon spectroscopy. Multiphoton absorption can also lead to the ionization of the molecule and its efficiency is strongly enhanced when the absorption of the photons is sequential, i.e. if it proceeds via a resonant intermediate state. Resonantly enhanced multiphoton ionization is a powerful, sensitive detection technique in molecular spectroscopy [2], particularly when it is combined with a mass sensitive detection of the ions [3]. The latter technique has been successfully applied to measure spectra of weakly bound van der Waals clusters [4-7] with vibrational resolution. For this purpose the mass selection is important since the 
spectrum of a particular cluster species has to be measured in a molecular beam containing a variety of clusters of different structure and size.

For determination of molecular structure it is necessary to obtain information on the rotational line structure of the electronic transitions. This requires high resolution in the sub-Doppler regime. At first sight, resonantly enhanced multiphoton ionization appears to be no suitable method for that purpose, since rapid excited state absorption from the intermediate state is needed for efficient ionization. This may lead to a shortening of the effective lifetime of the intermediate state and to a broadening of the spectra. In recent work we have shown that this broadening can be avoided and for the first time sub-Doppler spectra have been measured with resonantly enhanced two-photon ionization [8].

In this work we would like to illustrate the species selectivity of resonanceenhanced two-photon ionization for the extreme example of chemically identical isotopic van der Waals complexes of benzene $-{ }^{20} \mathrm{Ne}$ and benzene $-{ }^{22} \mathrm{Ne}$ present with natural abundance in the molecular beam. The rotationally resolved sub-Doppler spectra of both isotopic species will be presented and analyzed, yielding reliable van der Waals bond lengths for both isotopic species.

\section{EXPERIMENTAL}

The experimental set-up for the technique of resonance-enhanced two-photon ionization is similar to the one used previously to record rotationally resolved spectra of benzene-Ar [8]. Only a brief review will be given here, with particular emphasis on the experimental conditions of the benzene-Ne measurements.

The benzene-Ne clusters are formed in a skimmed supersonic jet expansion of a premixed gas sample of 40 mbar benzene and 5 bar neon in natural isotopic mixture $\left(90.5 \%{ }^{20} \mathrm{Ne}, 9.3 \%{ }^{22} \mathrm{Ne}\right)$. With a $50: 1$ collimation of the molecular beam the residual Doppler width in the interaction region is reduced to about $60 \mathrm{MHz}$ at the transition frequency of $38603 \mathrm{~cm}^{-1}$.

To excite the clusters to the $6^{1}$ vibration in the $\mathrm{S}_{1}\left({ }^{1} \mathrm{~B}_{2 \mathrm{u}}\right)$ electronic state, the light of a cw single-mode dye laser (Coherent 699 ) is pulsed amplified, frequency doubled in a BBO crystal and perpendicularly crossed with the supersonic jet. UV light pulses with a Fourier-transform limited bandwidth of $110 \mathrm{MHz}$ and an energy of up to $0.5 \mathrm{~mJ}$ are available to resolve most of the rotational structure and to easily saturate the rovibronic transitions under investigation. Ionization is achieved by the absorption of a second photon from a frequency doubled pulsed dye laser (FL 2002, Lambda Physik) with a frequency width of $0.5 \mathrm{~cm}^{-1}$ at $2740 \AA$, leading to an excess energy above the ionization potential of $700 \mathrm{~cm}^{-1}\left(1600 \mathrm{~cm}^{-1}\right)$ for the $6_{0}^{1}\left(6_{0}^{1} 1_{0}^{1}\right)$ transition. The ionizing laser beam collinearly overlaps with the excitation laser beam. The delay of the ionizing laser pulse is $2-3 \mathrm{~ns}$, so that both pulses effectively overlap 
in time. To avoid broadening of the spectra due to saturation of the first excitation step and strong and fast ionization by the second step, the laser intensities in the interaction region are reduced by attenuation and defocusing. No broadening was found at an intensity of $5 \times 10^{3} \mathrm{~W} \mathrm{~cm}^{-2}$ of the excitation laser and $2 \times 10^{6} \mathrm{~W} \mathrm{~cm}^{-2}$ of the ionizing laser.

The ions are accelerated perpendicular to the molecular beam and the laser beams in a Wiley-McLaren type home-built time-of-flight mass spectrometer [9]. The length of the drift region of $20 \mathrm{~cm}$ yields a maximum mass resolution of 300 , which was obtained by a second order correction according to ref. 10 . As demonstrated in the mass spectrum of Fig. 1 the masses of $\mathrm{C}_{6} \mathrm{H}_{6} \cdot{ }^{20} \mathrm{Ne}$ and $\mathrm{C}_{6} \mathrm{H}_{6} \cdot{ }^{22} \mathrm{Ne}(98$ and $100 \mathrm{u}$ ) are well separated. Thus the spectrum of both clusters can be measured simultaneously by integrating each mass peak in a gated boxcar integrator during the laser scan. If fluorescence were being used as the detection method, both spectra as well as the benzene and benzene dimer spectra would overlap, as all species are excited at the same wavelength (Fig. 1). To avoid saturation of the detector by the large number of $\mathrm{C}_{6} \mathrm{H}_{6}{ }^{+}$ions, which reduce the sensitivity of the channel plates for the masses of interest arriving at later times, we place an additional grid in front of the detector. A potential comparable to the accelerating voltage repels the saturating ions and is then switched off shortly before the cluster ions under investigation arrive at the grid.

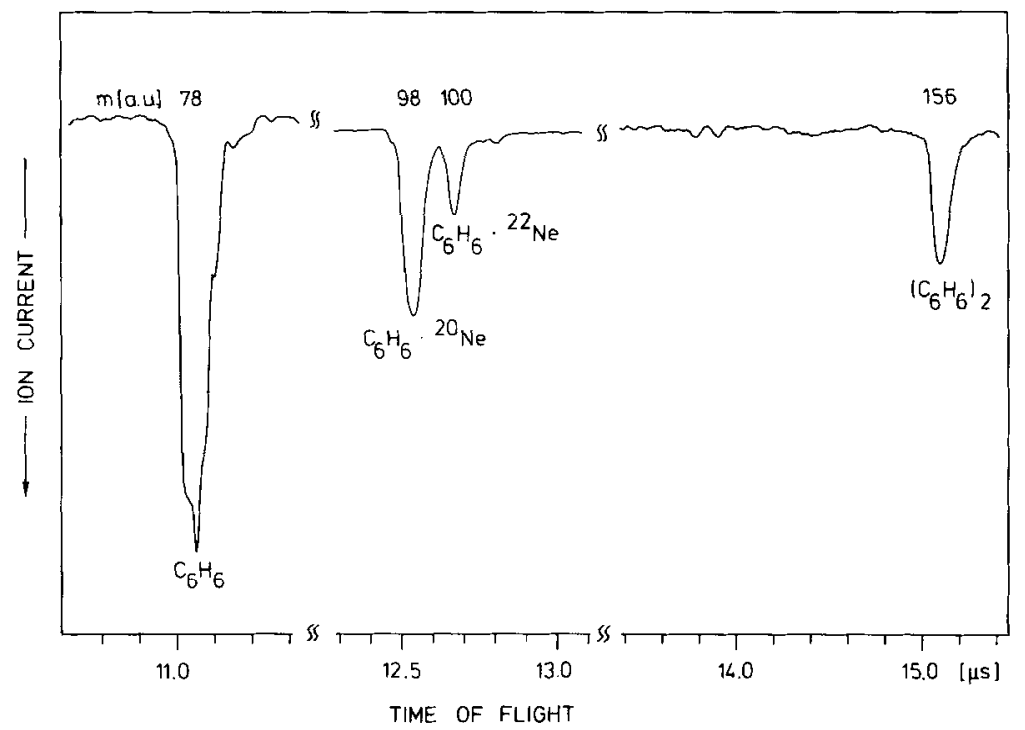

Fig. 1. Time-of-flight mass spectrum between 10.5 and $15.5 \mu$ s measured for an excitation energy of $38603.21 \mathrm{~cm}^{-1}$ (Q-subbranch $K^{\prime}=0, \Delta K=-1$ of the $\mathrm{C}_{6} \mathrm{H}_{6} \cdot$ Ne clusters). The mass peaks of the two isotopic clusters $\mathrm{C}_{6} \mathrm{H}_{6} \cdot{ }^{\circ 0} \mathrm{Ne}(98 \mathrm{u})$ and $\mathrm{C}_{6} \mathrm{H}_{6} \cdot{ }^{22} \mathrm{Ne}(100 \mathrm{u})$ are well separated. The strong signal of $\mathrm{C}_{6} \mathrm{H}_{6}{ }^{+}$ions at $11.1 \mu \mathrm{s}$ is not to scale due to saturation of the detection system. 
RESULTS

General appearance of the $6_{0}^{l}$ band

In Fig. 2 two highly resolved spectra around $38603 \mathrm{~cm}^{-1}$ are shown. This is the spectral range of the $6_{0}^{1}$ band of benzene-Ne which has been measured with vibrational resolution in the recent work of Stephenson and Rice [11]. The upper spectrum of Fig. 2 was obtained when ions with $100 \mathrm{u}$ were monitored. Here the vertical scaling is multiplied by a factor of 5 as compared to the lower spectrum which was obtained from ions with $98 \mathrm{u}$. The average intensity ratio of the lines in both spectra agrees very well with what would be expected from the isotopic abundance of ${ }^{20} \mathrm{Ne}(90.5 \%)$ and ${ }^{22} \mathrm{Ne}(9.3 \%)$. The structure of both spectra seems to be identical. However, there are noticeable small blue shifts of some marked lines at the high frequency side of the lower spectrum. The relative position of both spectra is very accurate $( \pm 10 \mathrm{MHz})$ as they were measured simultaneously in a single laser scan. On top of Fig. 2 the assignment of 5 lines in the R-branch with common $J^{\prime}=5$ but varying $K^{\prime}$ is indicated. It is seen that the isotopic shift is strongest for lines with high $J$ but low $K$. This is mainly due to the differing value of the $B$ rotational constant of both isotopes. $A$ does not depend on the mass of the added atom. For a given $J$ but

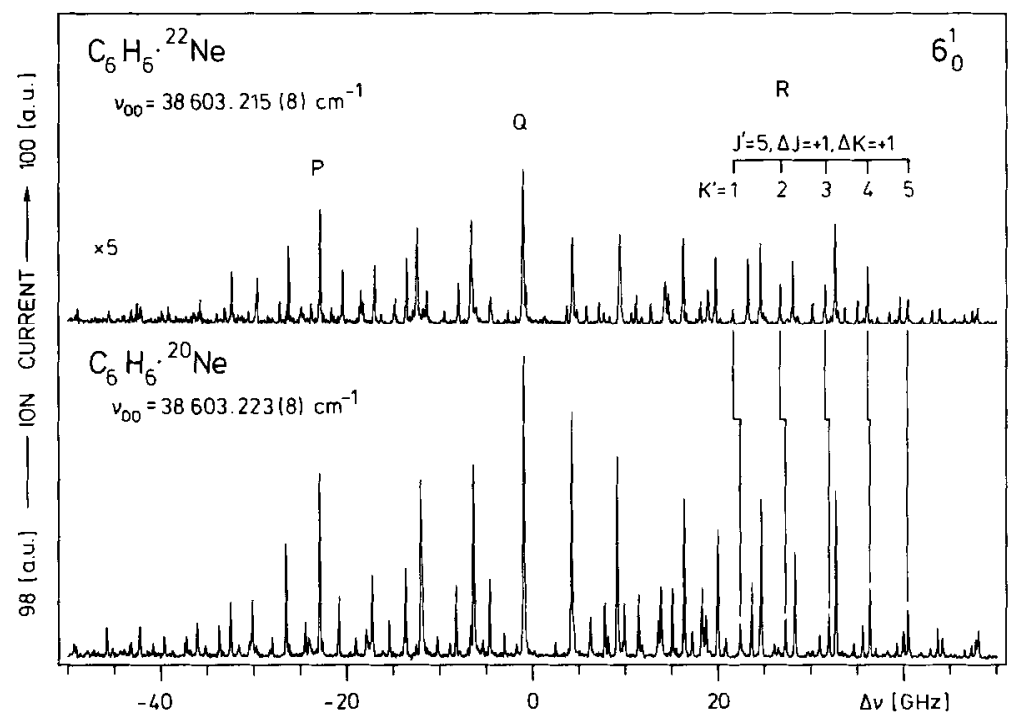

Fig. 2. Mass-selected resonance-enhanced two-photon ionization spectrum of the $6_{0}^{1}$ band of $\mathrm{C}_{6} \mathrm{H}_{6} \cdot{ }^{20} \mathrm{Ne}$ (lower trace) and $\mathrm{C}_{6} \mathrm{H}_{6} \cdot{ }^{22} \mathrm{Ne}$ (upper trace, magnified by a factor of 5 ) in natural isotopic abundance. The spectrum is divided in rotationally resolved $\mathrm{P}$ - and $\mathrm{R}$-branches in the red and blue part, and unresolved Q-subbranches leading to strong single peaks. Due to the different rotational constant $B$ of the two isotopic clusters the line positions indicated in the R-branch are different. 
increasing $K$ the isotope dependent part of the rotational energy $B J(J+1)$ is partly compensated by the $-B K^{2}$ term and the isotopically independent terms are left (see eqn. (2)).

The linewidth of the sharpest features in the wings of the vibronic band is $130 \mathrm{MHz}$. As will be shown below, in these parts of the vibronic band individual single rotational transitions are completely resolved. Their linewidth agrees with the value given by the convolution of the frequency width of the UV laser pulse $(110 \mathrm{MHz})$ and the Doppler width in the skimmed beam $(60 \mathrm{MHz})$. Hence no additional broadening due to fast intramolecular dynamic processes occurs in the $6^{1}$ state at an excess energy of $520 \mathrm{~cm}^{-1}$.

The general appearance of the vibronic band is similar to that of the same band in benzene-Ar presented in our recent work [8]. It is the spectrum of a prolate symmetric top and we conclude that the $\mathrm{Ne}$ atom is located on the $C_{6}$ axis above the benzene plane. Its gross ordering is illustrated in the upper spectrum of Fig. 2. The P- and R-branches consist of widely spaced lines, whereas the strong sharp features in the centre of the band belong to the $\mathrm{Q}$ branch and represent subbands of completely overlapping lines with constant $K^{\prime}$ and $\Delta K$ but varying $J^{\prime}$. This part of the band differs from the $6_{0}^{1}$ band in benzene-Ar, where the individual lines were partly resolved since they do not completely overlap and lead to blueshaded wings.

The total width of the $6_{0}^{1}$ band shown in Fig. 2 is only $100 \mathrm{GHz}$. This points to a lower rotational temperature than achieved for benzene-Ar $(\geqslant 2.3 \mathrm{~K})$. The temperature for the present experiment found from the analysis described below is $1.0-1.5 \mathrm{~K}$.

\section{Rotational analysis}

For a precise determination of the rotational constants a computer fit of the spectrum was performed according to the semi-rigid symmetric top energy formula for a transition to a degenerate vibration in the electronically excited state

$$
\begin{aligned}
E & =\Delta E_{\mathrm{rot}}+\nu_{00} \\
& =B_{\mathrm{v}}^{\prime} J^{\prime}\left(J^{\prime}+1\right)+\left(A_{\mathrm{v}}^{\prime}-B_{\mathrm{v}}^{\prime}\right) K^{\prime 2} \\
& -D_{J}^{\prime} J^{\prime 2}\left(J^{\prime}+1\right)^{2}-D_{J K}^{\prime} J^{\prime}\left(J^{\prime}+1\right) K^{\prime 2}-D_{K}^{\prime} K^{4} \pm 2 A_{\mathrm{v}}^{\prime} K^{\prime} \zeta_{\text {eff }}^{\prime} \\
& -B_{0}^{\prime \prime} J^{\prime \prime}\left(J^{\prime \prime}+1\right)-\left(A_{0}^{\prime \prime}-B_{0}^{\prime \prime}\right) K^{\prime 2} \\
& +D_{J}^{\prime \prime} J^{\prime \prime 2}\left(J^{\prime \prime}+1\right)^{2}+D_{J K}^{\prime \prime} J^{\prime \prime}\left(J^{\prime \prime}+1\right) K^{\prime \prime 2}+D_{K}^{\prime \prime} K^{\prime \prime 4}+\nu_{00}
\end{aligned}
$$

Global fits of all spectroscopic constants yield precise values for $\Delta B=B_{v}^{\prime}-$ $B_{0}^{\prime \prime}$, but are not sufficiently sensitive to the absolute values of $B_{0}^{\prime \prime}$ or $B_{\mathrm{v}}^{\prime}$. For this reason we first attempted to evaluate a reasonable value for $B_{0}^{\prime \prime}$. Using the 
method of combination differences we are able to calculate transition frequencies between rotational states in the ground state from our electronic spectra. By subtracting the frequencies of transitions which belong to the same excited state (same $J^{\prime}, K^{\prime}$ and $\Delta K$, but different $\Delta J$ ) the rotational constants of the excited state are eliminated and the ground state constants can be directly determined from the differences. According to the $\Delta J=0, \pm 1$ selection rule (Q,R,P transition) the following three combination differences exist for each excited state, not more than two of which are used to avoid redundance:

$$
\begin{aligned}
E_{\mathrm{R}}-E_{\mathrm{Q}}= & 2 B_{0}^{\prime \prime} J^{\prime}-4 D_{J}^{\prime \prime} J^{\prime 3}-2 D_{J K}^{\prime \prime} J^{\prime}\left(K^{\prime}-\Delta K\right)^{2} \\
E_{\mathrm{Q}}-E_{\mathrm{P}}= & 2 B_{0}^{\prime \prime}\left(J^{\prime}+1\right)-4 D_{J}^{\prime \prime}\left(J^{\prime}+1\right)^{3}-2 D_{J K}^{\prime \prime}\left(J^{\prime}+1\right)\left(K^{\prime}-\Delta K\right)^{2} \\
E_{\mathrm{R}}-E_{\mathrm{P}}= & 2 B_{0}^{\prime \prime}\left(2 J^{\prime}+1\right)-4 D_{J}^{\prime \prime}\left[\left(J^{\prime}+1\right)^{3}+J^{\prime 3}\right] \\
& -2 D_{J K}^{\prime \prime}\left(2 J^{\prime}+1\right)\left(K^{\prime}-\Delta K\right)^{2}
\end{aligned}
$$

The $B_{0}^{\prime \prime}$ value is obtained by a least-squares fit to the combination differences observed in the spectrum. $D_{J}^{\prime \prime}$ and $D_{J K}^{\prime \prime}$ are constrained to zero, since the spectral resolution was not high enough to yield significant values for centrifugal distortion. In a second step the rotational constants $A_{\mathrm{v}}^{\prime}, B_{\mathrm{v}}^{\prime}$ and $\zeta_{\text {eff }}^{\prime}$ of the excited state are fitted with fixed $B_{0}^{\prime \prime}$ and the additional assumption is made that the bond lengths in benzene are not changed by the addition of a $\mathrm{Ne}$ atom. This implies that $A_{0}^{\prime \prime}$ of the cluster is equal to $C_{0}^{\prime \prime}$ of benzene.

The rotational constants of $\mathrm{C}_{6} \mathrm{H}_{6}{ }^{\circ 2} \mathrm{Ne}\left(\mathrm{C}_{6} \mathrm{H}_{6}{ }^{\circ 2} \mathrm{Ne}\right)$ from a fit to 74 (67) observed line positions are listed in Table 1. The standard deviation is 18 (23) $\mathrm{MHz}$. It is interesting to see that there is only a small change of $10^{-4} \mathrm{~cm}^{-1}$ of the rotational constant $B$ between the electronic ground state and the electronically excited $6^{1}$ state. This is much less than the change of $A$ which amounts to $4 \times 10^{-3} \mathrm{~cm}^{-1}$. For $A_{0}^{\prime \prime}$ assumed to be identical to $C_{0}^{\prime \prime}$ of $\mathrm{C}_{6} \mathrm{H}_{6}$ (see above) we obtain an $A_{\mathrm{v}}^{\prime}$ value which differs only by $8 \times 10^{-5} \mathrm{~cm}^{-1}$ from the $C_{\mathrm{v}}^{\prime}$ value of benzene. Though the agreement is not as perfect as in benzene-Ar $\left(3 \times 10^{-6}\right.$ $\mathrm{cm}^{-1}$ ) [8], this result corroborates the assumption for the $A_{0}^{\prime \prime}$ ground state constant. A possible reason for the incomplete agreement of $A_{0}^{\prime \prime}$ and $A_{\mathrm{v}}^{\prime}$ may be the relatively strong deviation of the $\zeta_{\text {eff }}^{\prime}$ value of the benzene-Ne cluster from that of benzene. The appropriate values for the $6_{0}^{1}$ band of $\mathrm{C}_{6} \mathrm{H}_{6}$ are included in Table 1.

The same fitting procedure was applied to the $6_{0}^{1} 1_{0}^{1}$ bands of both $\mathrm{C}_{6} \mathrm{H}_{6}{ }^{20} \mathrm{Ne}$ and $\mathrm{C}_{6} \mathrm{H}_{6}{ }^{22} \mathrm{Ne}$ and the resulting rotational constants are summarized in Table 1. For illustration of the quality of the fit, in Fig. 3 the experimental spectrum of the $6_{0}^{1} 1_{0}^{1}$ band of $\mathrm{C}_{6} \mathrm{H}_{6}{ }^{\cdot 20} \mathrm{Ne}$ is compared with the theoretical spectrum. It was calculated from the spectroscopic constants and, in order to simulate the spectral resolution in the experiment, the stick spectrum was convoluted with a gaussian linewidth of $130 \mathrm{MHz}$ (FWHM) corresponding to the experimental linewidth in the $6_{0}^{1}$ band. There is an excellent agreement of the theoretical and experimental line positions demonstrating the accuracy of the fitted ro- 


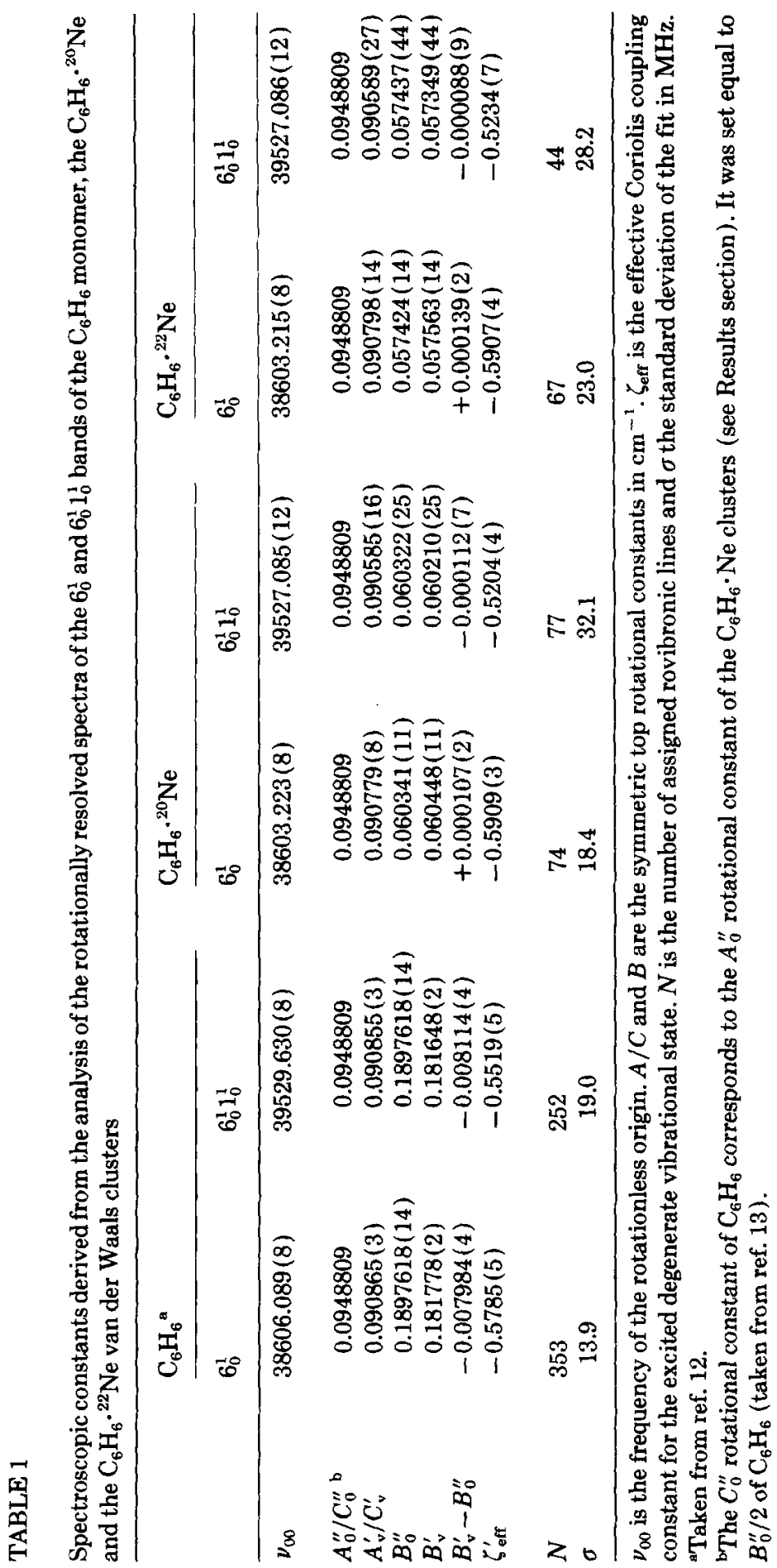




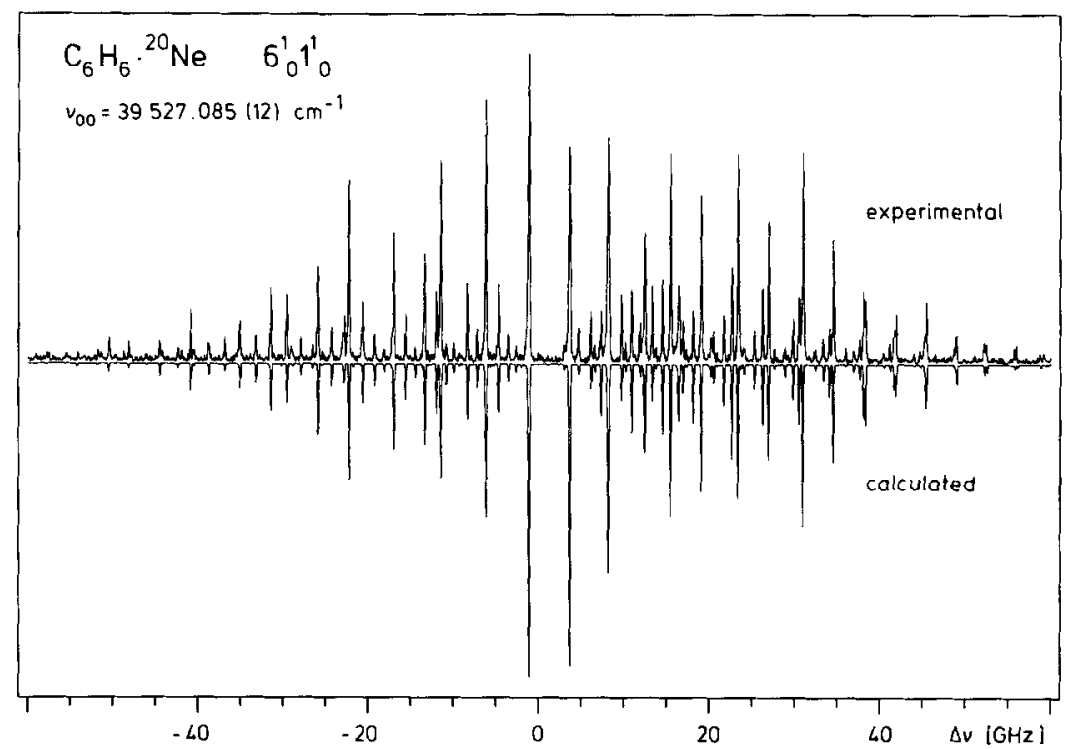

Fig. 3. Comparison of the resonance-enhanced two-photon ionization spectrum of the $6_{0}^{1} 1_{0}^{1}$ band of $\mathrm{C}_{6} \mathrm{H}_{6}{ }^{.0} \mathrm{Ne}$ (upper trace) with a theoretical spectrum (lower trace) calculated with the rotational constants of Table 1 , which were evaluated from a computer fit to the observed line positions. The theoretical stick spectrum was calculated with a rotational temperature of $1.3 \mathrm{~K}$ and convoluted with a gaussian linewidth of $130 \mathrm{MHz}$ (FWHM).

tational constants. No asymmetry splitting of lines with high $J$ and small $K$ is seen in the experimental spectrum indicating that benzene-Ne is, to a high precision, a symmetric top. The linewidth in the experimental spectrum of 160 $\mathrm{MHz}$ is somewhat larger than in the theoretical spectrum. This is broader than the linewidth of $130 \mathrm{MHz}$ in the experimental spectrum of the $6_{0}^{1}$ band shown in Fig. 2. Its origin could be due to a fast relaxation process occurring at the higher excess energy of $1440 \mathrm{~cm}^{-1}$ of the $6^{1} 1^{1}$ state. In our recent experiments on benzene-Ar [8] no broadening of the $6_{0}^{1} 1_{0}^{1}$ band was found. A priori it is not clear why the dynamic behavior of benzene-Ne and benzene-Ar should be different at the same excess energy. More likely, a slightly broader laser linewidth is responsible for this effect. Unfortunately, the laser linewidth was not checked for this particular wavelength.

\section{Vibrational analysis}

The rotational analysis described in the previous section yields accurate values for the frequencies $\nu_{00}$ of the band origins. The absolute values for $\nu_{00}$ have been found by comparison with the simultaneously measured iodine absorption spectrum whose transition frequencies are known with high precision [14]. The difference $\Delta \nu_{00}=\nu_{00}\left({ }^{20} \mathrm{Ne}\right)-\nu_{00}\left({ }^{22} \mathrm{Ne}\right)$ of the band origins of both iso- 
topic clusters can be determined with much higher accuracy, since the spectra were measured simultaneously as described earlier. For the $6_{0}^{1}\left(6_{0}^{1} 1_{0}^{1}\right)$ transition we find $\Delta \nu_{00}=0.0077(-0.0009) \mathrm{cm}^{-1}$.

The values of $\nu_{00}$ for the two vibronic bands of benzene-Ne and of benzene are listed in Table 1. The red-shift of the $6_{0}^{1}$ band in $\mathrm{C}_{6} \mathrm{H}_{6} \cdot{ }^{20} \mathrm{Ne}\left(\mathrm{C}_{6} \mathrm{H}_{6} \cdot{ }^{\cdot 22} \mathrm{Ne}\right)$ is $2.866(2.874) \mathrm{cm}^{-1}$ and the red shift of the $6_{0}^{1} 1_{0}^{1}$ band is $2.545(2.544) \mathrm{cm}^{-1}$. There is a negligible small isotope effect in the red shift of both bands. However, similar to benzene-Ar [8], the difference of the $6_{0}^{1}$ and $6_{0}^{1} 1_{0}^{1}$ band origins is increased by $0.321(0.330) \mathrm{cm}^{-1}$ in the $\mathrm{C}_{6} \mathrm{H}_{6} \cdot{ }^{20} \mathrm{Ne}\left(\mathrm{C}_{6} \mathrm{H}_{6} \cdot{ }^{22} \mathrm{Ne}\right)$ cluster.

Structure of the benzene-Ne cluster

Since no asymmetry splitting is observed in the rotationally resolved spectra of the benzene-Ne cluster it is clear that the sixfold rotational axis of benzene is preserved in the cluster and the $\mathrm{Ne}$ atom is located on this axis. For this geometry and under the assumption that the bond lengths in the benzene ring are not changed in the cluster, a value for the benzene-Ne distance can be determined from the rotational constant $B$ in the cluster. This was accomplished with the procedure described in our recent work using the published bond lengths $r_{\mathrm{CC}}$ and $r_{\mathrm{CH}}$ of benzene [8]. A van der Waals bond length was calculated from the measured rotational constants $B_{0}^{\prime \prime}$ and $B_{\mathrm{v}}^{\prime}$ yielding average bond lengths in the electronic ground and the excited electronic state, respectively. The accuracy of these values can be checked by comparison of the two independent results for the $\mathrm{C}_{6} \mathrm{H}_{6} \cdot{ }^{\cdot 0} \mathrm{Ne}$ and $\mathrm{C}_{6} \mathrm{H}_{6} \cdot{ }^{22} \mathrm{Ne}$ clusters. The results given in Table 2 and the changes of $\left\langle r_{\mathrm{Ne}}\right\rangle$ with isotopic substitution, vibrational quantum number and electronic excitation can be summarized.

(i) The respective values for the average bond lengths in the ground $\left(\mathrm{S}_{0}\right)$ and excited $\left(\mathrm{S}_{1}\right)$ state, $\left\langle r_{\mathrm{Ne}}^{\prime \prime}\right\rangle$ and $\left\langle r_{\mathrm{Ne}}^{\prime}\right\rangle$, differ by 5-7 $\mathrm{m} \AA$ for the two iso-

\section{TABLE 2}

Average bond lengths $\left\langle r_{\mathrm{Ne}}\right\rangle$ (distance of the Ne atom from the plane of the benzene ring) in the $\mathrm{C}_{6} \mathrm{H}_{6} \cdot \mathrm{Ne}$ van der Waals clusters, determined from the rotational constants of Table 1 . The errors represent the uncertainty of $\left\langle r_{\mathrm{Ne}}\right\rangle$ due to the uncertainty of the rotational constants and the $\left\langle r_{\mathrm{CC}}\right\rangle,\left\langle r_{\mathrm{CH}}\right\rangle$ distances of ref. 8

\begin{tabular}{|c|c|c|c|}
\hline & & $\begin{array}{c}\left\langle r_{\mathrm{Ne}}\right\rangle\left({ }^{20} \mathrm{Ne}\right) \\
{[\AA]}\end{array}$ & $\begin{array}{c}\left\langle r_{\mathrm{Ne}}\right\rangle\left({ }^{22} \mathrm{Ne}\right) \\
{[\AA]}\end{array}$ \\
\hline $\mathbf{S}_{0}$ & $\begin{array}{l}6_{0}^{1} \\
6_{0}^{1} 1_{0}^{1}\end{array}$ & $\begin{array}{l}3.460(1) \\
3.461(1)\end{array}$ & $\begin{array}{l}3.454(1) \\
3.454(2)\end{array}$ \\
\hline $\mathrm{S}_{1}$ & $\begin{array}{l}6_{0}^{1} \\
6_{0}^{1} 1_{0}^{1}\end{array}$ & $\begin{array}{l}3.420(3) \\
3.430(3)\end{array}$ & $\begin{array}{l}3.415(3) \\
3.424(4)\end{array}$ \\
\hline
\end{tabular}


topes. This is larger than the errors of $\left\langle r_{\mathrm{Ne}}\right\rangle$ resulting from the inaccuracy of the rotational constants and of the $r_{\mathrm{CC}}$ and $r_{\mathrm{CH}}$ values and will be discussed in more detail (see below).

(ii) The additional excitation of one quantum of the totally symmetric C-C stretch vibration in the electronically excited $S_{1}$ state of benzene leads to a 10 $m \AA$ increase of the average van der Waals bond length $\left\langle r_{N_{e}}^{\prime}\right\rangle$. A nearly identical increase of $\left\langle r_{\mathrm{Ne}}^{\prime}\right\rangle$ is observed for both isotopes. It is produced by the alteration of $B_{\mathrm{v}}^{\prime}$ with excitation (see Table 1) of the benzene vibration due to harmonic, anharmonic and vibration-rotation coupling contributions.

(iii) Electronic excitation to the $6^{1}$ state results in a decrease of the average bond length of $40 \mathrm{~mA}$ for both isotopes. This is a relatively small change, demonstrating that the van der Waals forces between the benzene and the Ne atom do not change very much if the benzene molecule is electronically excited.

\section{DISCUSSION}

The results of the previous section are now discussed and compared with the recent results of benzene-Ar.

The average van der Waals benzene-Ne bond length of the two isotopes $\mathrm{C}_{6} \mathrm{H}_{6} \cdot{ }^{\circ 0} \mathrm{Ne}$ and $\mathrm{C}_{6} \mathrm{H}_{6} \cdot{ }^{22} \mathrm{Ne}$ differ by $6 \mathrm{m \AA}$, although the equilibrium distance is expected to be the same in both isotopic clusters. Hence, we conclude that the $\left\langle r_{\mathrm{Ne}}\right\rangle$ values are influenced by the slightly different frequencies of the van der Waals modes in $\mathrm{C}_{6} \mathrm{H}_{6} \cdot{ }^{20} \mathrm{Ne}$ and $\mathrm{C}_{6} \mathrm{H}_{6} \cdot{ }^{22} \mathrm{Ne}$. Different frequencies lead to differing zero-point vibrations and thus to different contributions to the rotational constant $B_{0}^{\prime \prime}$ and $B_{\mathrm{v}}^{\prime}$ of the two isotopic clusters. For the two isotopically substituted benzene-Ar clusters, $\mathrm{C}_{6} \mathrm{H}_{6} \cdot \mathrm{Ar}$ and $\mathrm{C}_{6} \mathrm{D}_{6} \cdot \mathrm{Ar}$ we found that $\left\langle r_{\mathrm{Ar}}\right\rangle$ in $\mathrm{C}_{6} \mathrm{D}_{6} \cdot \mathrm{Ar}$ is smaller by only $3 \mathrm{~m} \AA$ than in $\mathrm{C}_{6} \mathrm{H}_{6} \cdot \mathrm{Ar}$. This smaller effect in isotopically substituted benzene-Ar can be qualitatively understood if we consider the relative reduced mass change of both species in the diatomic approximation. The isotopic substitution of all $\mathrm{H}$ atoms in $\mathrm{C}_{6} \mathrm{H}_{6} \cdot \mathrm{Ar}$ by $\mathrm{D}$ atoms given rise to a $2.5 \%$ mass change whereas the reduced mass change is by a factor of 3 larger (7.8\%) when ${ }^{20} \mathrm{Ne}$ is replaced by ${ }^{22} \mathrm{Ne}$ in $\mathrm{C}_{6} \mathrm{H}_{6} \cdot \mathrm{Ne}$.

The influence of the large amplitude motion of the van der Waals vibrations has to be taken into account when information on the equilibrium distance is deduced from the experimentally determined average van der Waals bond length. For this reason absolute values $\left\langle r_{\mathrm{Ne}}\right\rangle$ of the benzene-Ne bond length cannot be directly interpreted as equilibrium distances. Both values could differ by several $10 \mathrm{~m} \AA$. Smaller discrepancies are expected for the differences of the average van der Waals bond length $\left\langle r_{\mathrm{Ne}}\right\rangle$ between the electronic ground $\left(S_{0}\right)$ and excited $\left(S_{1}\right)$ state. Provided the van der Waals potentials do not change in both electronic states we can assume that $r_{\mathrm{eq}}$ and $\left\langle r_{\mathrm{Ne}}\right\rangle$ differ by the 
same amount in both states due to the equal contributions of large amplitude vibrations. This condition seems to be fulfilled in benzene-Ne since the $6_{0}^{1}$ transition is shifted only by $2.86 \mathrm{~cm}^{-1}$ from the $6_{0}^{1}$ transition in benzene. Hence the bond length change upon electronic excitation $r_{\text {eq }}^{\prime}-r_{\text {eq }}^{\prime \prime}$ is given to a good approximation by $\left\langle r_{\mathrm{Ne}}^{\prime}\right\rangle-\left\langle r_{\mathrm{Ne}}^{\prime \prime}\right\rangle$. We found a value of $-40(-30) \mathrm{m} \AA$ for the $6_{0}^{1}\left(6_{0}^{1} 1_{0}^{1}\right)$ transition. This compares well with the slightly larger value of $-60(-58) \mathrm{mA}$ in benzene-Ar.

\section{SUMMARY AND CONCLUSION}

In this work we have shown that rotationally resolved spectra of the $6_{0}^{1}$ and $6{ }_{0}^{1} 1_{0}^{1}$ bands in the two isotopic clusters $\mathrm{C}_{6} \mathrm{H}_{6} \cdot{ }^{\cdot 20} \mathrm{Ne}$ and $\mathrm{C}_{6} \mathrm{H}_{6} \cdot{ }^{22} \mathrm{Ne}$ can be measured with the technique of resonance enhanced two-photon ionization. The high resolution was feasible by application of the pulsed amplified and frequency doubled light of a cw dye laser with a frequency width of $110 \mathrm{MHz}$ and by reduction of the Doppler width to $60 \mathrm{MHz}$ in a skimmed supersonic jet. Multiphoton ionization in combination with mass selective ion detection enabled us to measure both isotopic clusters selectively within their natural mixture $\left({ }^{20} \mathrm{Ne} /{ }^{22} \mathrm{Ne}=90: 10\right)$. The analysis of these sp $\epsilon$ ira yields accurate rotational constants of the cluster in the electronic ground state and for the electronically excited state when the $6^{1}$ and the $6^{1} 1^{1}$ vibronic states are excited. From these constants an average van der Waals bond length of $3.46 \AA$ in the electronic ground state is calculated. The van der Waals bond length decreases by $40 \mathrm{~mA}$ when benzene is electronically excited.

The rotational analysis reveals that the rotational constants are significantly affected by the large amplitude van der Waals vibrations of the cluster. This becomes obvious, e.g. from the differing average van der Waals bond lengths found for the two isotopic clusters. For $\mathrm{C}_{6} \mathrm{H}_{6}{ }^{20} \mathrm{Ne}$ and $\mathrm{C}_{6} \mathrm{H}_{6}{ }^{22} \mathrm{Ne}$ we expect the same equilibrium bond length, but from the rotational constants we find a difference of $6 \mathrm{~m} \AA$. The influence of the large amplitude van der Waals motion on the rotational constants could be treated in a quantitative way if the van der Waals potentials of the bending and the stretching mode were known. For this, measurements of the van der Waals bands with rotational resolution would be helpful. These experiments are in progress in our laboratory.

\section{ACKNOWLEDGMENTS}

The authors thank R. Sußmann for experimental help and A.M. Smith for assistance during the assignment of the spectra. Financial support from the Deutsche Forschungsgemeinschaft and from the Fonds der Chemischen Industrie is gratefully acknowledged. 


\section{REFERENCES}

1 S.H. Lin, Y. Fujimura, H.J. Neusser and E.W. Schlag, Multiphoton Spectroscopy of Molecules, Academic Press, Orlando, 1984.

2 P.M. Johnson and C.E. Otis, Ann. Rev. Phys. Chem., 32 (1981) 139.

P.M. Johnson, J. Chem. Phys., 62 (1975) 4562.

3 U. Boesl, H.J. Neusser and E.W. Schlag, Z. Naturforsch. Teil A, 33 (1978) 1546.

4 J.B. Hopkins, D.E. Powers and R.E. Smalley, J. Phys. Chem., 85 (1981) 3739.

5 S. Leutwyler, U. Even and J. Jortner, Chem. Phys. Lett., 86 (1982) 439.

6 K.H. Fung, H.L. Selzle and E.W. Schlag, J. Phys. Chem., 87 (1983) 5113.

7 K.S. Law, M. Schauer and E.R. Bernstein, J. Chem. Phys., 81 (1984) 4871.

8 Th. Weber, A. von Bargen, E. Riedle and H.J. Neusser, J. Chem. Phys., 92 (1990) 90.

9 W.C. Wiley and I.H. McLaren, Rev. Sci. Instrum., 26 (1955) 1150.

10 R. Weinkauf, K. Walter, C. Weickhardt, U. Boesl and E.W. Schlag, Z. Naturforsch. Teil A, 44 (1989) 1219.

11 T.A. Stephenson and S.A. Rice, J. Chem. Phys., 81 (1984) 1083.

12 E. Riedle, Th. Knittel, Th. Weber and H.J. Neusser, J. Chem. Phys., 91 (1989) 4555.

13 J. Pliva and A.S. Pine, J. Mol. Spectrosc., 93 (1982) 209.

14 S. Gerstenkorn and P. Luc, Atlas du spectre d'absorption de la molecule de l'iode, CNRS, Paris, 1978. A correction of $-0.0056 \mathrm{~cm}^{-1}$ was used according to $S$. Gerstenkorn and P. Luc, Rev. Phys. Appl., 14 (1979) 791. 